\title{
Fasting remnant lipoproteins can predict postprandial hyperlipidemia
}

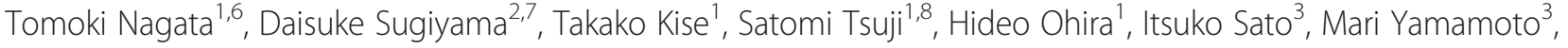
Hitomi Kohsaka ${ }^{3}$, Seiji Kawano ${ }^{3}$, Shizuya Yamashita ${ }^{4}$, Yuichi Ishikawa ${ }^{5}$ and Yoshio Fujioka ${ }^{{ }^{*}}$

\begin{abstract}
Background: Hypertriglyceridemia and postprandial hyperlipidemia is thought to play an important role in atherosclerosis, but to select patients at high-risk for cardiovascular diseases is difficult with triglycerides (TG) alone in these patients.

Methods: To predict postprandial hyperlipidemia without inconvenient test meal loading, we examined lipid concentrations before and after test meal loading and fasting adiponectin, and investigated which of these other than TG were significant during the fasting period in 45 healthy individuals (men: women, 26:19).

Results: TG, remnant-like particle-cholesterol and -triglyceride (RemL-C, RLP-C, and RLP-TG), and TG/apolipoprotein (аро)B were significantly elevated after loading and fasting values significantly and positively correlated with incremental area under the curve (iAUC) $(r=0.80, r=0.79, r=0.63, r=0.58, r=0.54 ; p<0.0001)$. Fasting adiponectin positively correlated with fasting high-density lipoprotein-cholesterol $(r=0.43, p<0.005)$ and apoA-I $(r=0.34, p<0.05)$, and negatively correlated with iAUC of TG, RemL-C, RLP-C, RLP-TG, and TG/apoB ( $r=-0.37, r=-0.41, r=-0.37, r=-0.36$, $r=-0.37 ; p<0.05$ ). We constructed the model of multivariable linear regression analysis without fasting $\mathrm{TG}$. In the sex-, BMI-, age-, and waist circumference-adjusted analysis of postprandial TG elevation $2 \mathrm{~h}$ after test meal loading in all participants, RemL-C, RLP-C, RLP-TG, and TG/apoB were significant factors, but adiponectin was not.

Conclusion: Fasting triglyceride-rich lipoprotein-related values, especially RemL-C, RLP-C, RLP-TG, and TG/apoB are useful predictors of postprandial hyperlipidemia in young healthy individuals. Although fasting adiponectin concentration correlated with the iAUCs for TG, RemL-C, RLP-C, RLP-TG, and TG/apoB, it was not a significant predictor of postprandial hyperlipidemia in multivariable linear regression analysis.
\end{abstract}

Keywords: Remnant lipoprotein, Postprandial hyperlipidemia, Triglyceride, Apolipoprotein B, Adiponectin.

\section{Background}

Epidemiological studies have recently shown that hypertriglyceridemia is associated with atherosclerosis, but the independence of the serum triglycerides (TG) concentration as a causal factor in promoting cardiovascular diseases (CVD) remains debatable and to select patients at high-risk for CVD is difficult with TG alone [1-3]. Individuals with mild hypertriglyceridemia without other metabolic disorders or severe hypertriglyceridemia such as primary chylomicronemia rarely have CVD.

\footnotetext{
*Correspondence: fujioka@nutr.kobegakuin.ac.jp

'Division of Clinical Nutrition, Faculty of Nutrition, Kobe Gakuin University, 518 Arise, Ikawadani-cho, Nishi-ku, Kobe 651-2180, Japan

Full list of author information is available at the end of the article
}

Postprandial hyperlipidemia is thought to play an important role in atherosclerosis, and concentrations of non-fasting TG are superior to those of fasting TG for predicting CVD [4-7]. Many studies have revealed that triglyceride-rich lipoproteins (TRL), especially chylomicron and very-low-density lipoprotein (VLDL) remnants, are atherogenic and that delayed removal of chylomicron remnants from the bloodstream induces postprandial hyperlipidemia [8-10]. However, screening large numbers of individuals using fat loading tests is inconvenient and neither a definition nor a standard method for predicting postprandial hyperlipidemia besides postprandial TG elevation has been established. These circumstances present a challenge in terms of how to distinguish patients at high-risk of CVD based

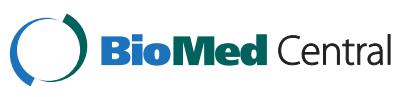


on fasting blood samples. We reported that fasting serum concentrations of remnant lipoproteins and apolipoprotein B-48 (apoB-48) besides TG might indicate postprandial hyperlipidemia even among normolipidemic individuals[11].

Adipocytes secrete adiponectin, which is a 224-aminoacid plasma protein [12,13]. Serum adiponectin concentrations are paradoxically reduced in individuals with a large visceral fat mass, and adiponectin plays a significant role in glucose and lipid metabolism [13-15]. However, few studies have examined the relationship between serum adiponectin concentrations and postprandial hyperlipidemia. Rubin et al. demonstrated that postprandial TG concentrations correlate with fasting adiponectin concentrations in 45 - 65-year-old individuals, including those with metabolic syndrome [16]. Maruyama et al. reported that serum concentrations of high molecular weight (HMW) adiponectin are associated with those of TG and remnant-like particle-triglyceride (RLP-TG) in individuals with type 1 diabetes before and after test meal loading [17].

Thus, the significance of adiponectin in postprandial hyperlipidemia needs to be clarified. Here, we used multivariable linear regression analysis to identify which factors among remnant lipoproteins, adiponectin and other particles during fasting are significant for predicting postprandial hyperlipidemia.

\section{Methods}

\section{Participants and physical examination}

We recruited 45 healthy individuals (men/women, 26/19) who had never been treated for diseases or taken drugs for at least 3 months prior to the study. We used data from 24 participants that we had previously analyzed [11] and we added another 21 individuals for the present study. All of the recruits provided written, informed consent to participate in the study. The institutional ethics committee of Kobe Gakuin University (HEB080701-1) approved the study protocol, which proceeded according to the Declaration of Helsinki. We measured the waist circumference and computed the body mass index (BMI) of all participants by dividing body weight by the square of the height $\left(\mathrm{kg} / \mathrm{m}^{2}\right)$.

\section{Study protocol}

The study proceeded as previously described [11]. Test meal A was developed by the Japanese Diabetes Society to assess both postprandial hyperglycemia and hyperlipidemia. This meal consisted of cream of chicken soup, a biscuit and custard pudding. The total $450 \mathrm{kcal}$ of energy was derived from $57.6 \mathrm{~g}$ of carbohydrate $(51.4 \%$ of the energy balance), $17.2 \mathrm{~g}$ of protein (15.3\%), and $16.6 \mathrm{~g}$ of fat (33.3\%), which is a slightly higher ratio of fat than that found in a typical Japanese breakfast (20\% - 25\%). Blood samples were obtained between 9 and 10 AM after $12 \mathrm{~h}$ of fasting and at 1, 2, 4, 6 and $8 \mathrm{~h}$ after test meal loading.

\section{Blood analysis}

Serum concentrations of HMW adiponectin and plasmaoxidized low-density lipoproteins (LDL) were determined using sandwich enzyme-linked immunosorbent assays (ELISA) (Fujirebio, Tokyo, Japan and Kyowa Medex, Tokyo, Japan, respectively). Serum concentrations of TG, total cholesterol, high-density lipoprotein-cholesterol (HDL-C) were determined enzymatically (Kyowa Medex, Tokyo, Japan). For low-density lipoprotein-cholesterol (LDL-C), we used the commercial kit by the selective solubilization method (Determiner L LDL-C, Kyowa Medex) because it is impossible to use Friedewald's formula in the postprandial cases. Serum concentrations of apoA-I, apoA-II, apoB, apoC-II, apoC-III, and apoE were measured using turbidimetric immunoassays (Nittobo, Tokyo, Japan). Serum small dense LDLcholesterol (sd-LDL-C) concentrations were measured using a precipitation method (Denka Seiken, Tokyo, Japan) [18]; serum concentrations of remnant-like particle-cholesterol (RLP-C) and RLP-TG were determined using immune adsorption (JIMRO II, Otsuka Pharmaceutical, Tokyo, Japan) [19]; serum remnant lipoprotein cholesterol (RemL-C) concentrations were measured using a homogeneous assay (MetaboLead RemL-C, Kyowa Medex) [20]. Serum high-sensitivity $\mathrm{C}$-reactive protein (hs-CRP) concentrations were determined using nephelometry (Dade Behring, Deerfield, IL, USA). Plasma glucose concentrations were determined enzymatically. Glycosylated hemoglobin A1c (HbA1c) values were determined using HPLC. Serum insulin concentrations were measured using an enzyme immunoassay. Non-HDL-C concentrations were computed by subtracting the value of $\mathrm{HDL}-\mathrm{C}$ from that of TC.

\section{Statistical analysis}

Values are expressed as means $\pm \mathrm{SD}$. Data below the threshold of RLP-C $(<2.0 \mathrm{mg} / \mathrm{dl})$ or RLP-TG $(<15 \mathrm{mg} / \mathrm{dL})$ were treated as 2.0 or $15 \mathrm{mg} / \mathrm{dL}$, respectively. TG, RemLC, RLP-C, RLP-TG, TG/apoB, and adiponectin were transformed into logarithmic values. The statistical significance of the data was evaluated using Welch's $t$-test or repeated ANOVA with Dunnett's test. Postprandial changes in values of TG, RemL-C, RLP-C, RLP-TG, or TG/apoB were quantified by calculating the incremental area under the curve (iAUC), which was estimated as the difference between the area defined below the baseline concentration and the area under the curve in each factor or parameter from $1 \mathrm{~h}$ to $8 \mathrm{~h}$ after test meal loading. 
Correlations between adiponectin and lipids as well as other factors were calculated using the formula for Pearson's correlation coefficient.

Independent predictors of TG at $2 \mathrm{~h}$ after test meal loading were identified by multivariable linear regression analysis adjusted with sex, BMI, age, waist circumference. Candidate predictors (RemL-C, RLP-C, RLP-TG, TG/apoB, and adiponectin) were analyzed separately because of correlation among candidate predictors. The purpose of our study was to elucidate the factors that predict postprandial hyperlipidemia besides TG, because it is difficult to select high-risk patients with TG alone. Therefore, we did not investigate fasting TG as candidate predictor in regression model. Data were statistically analyzed using SPSS Statistics 17.0 (IBM, Somers, NY, USA) and R version 2.12.1 (R Foundation for Statistical Computing, Vienna, Austria). Two-tailed values of $p<0.05$ were considered statistically significant.

\section{Results}

\section{Characteristics of subjects}

Table 1 shows the characteristics of the 26 men and 19 women participants, except for LPL mass (total 21; 15 men and 6 women). Although all factors were within normal limits, the values of height, body weight, waist circumference, and hs-CRP concentration were higher in men than in women. On the other hand, adiponectin concentration was higher in women than in men.

\section{Fasting and postprandial concentrations of lipids and their parameters}

Tables 2A (all participants) shows the changes of lipid, glucose, and insulin concentrations before and after test meal loading. Since some metabolic factors in Table 1

Table 1 Baseline characteristics of study participants

\begin{tabular}{lllll}
\hline & & Total & Men & Women \\
\hline Sex & $(\mathrm{n})$ & 45 & 26 & 19 \\
Age & (years) & $21.5 \pm 1.3$ & $21.4 \pm 1.5$ & $21.7 \pm 0.9$ \\
Height & $(\mathrm{m})$ & $1.66 \pm 0.08$ & $1.70 \pm 0.06$ & $1.61 \pm 0.07^{*}$ \\
Body weight & $(\mathrm{kg})$ & $58.1 \pm 8.8$ & $61.8 \pm 9.3$ & $53.1 \pm 4.9^{\dagger}$ \\
Waist circumference & $(\mathrm{cm})$ & $73.8 \pm 7.8$ & $73.5 \pm 7.5$ & $67.9 \pm 5.44^{\ddagger}$ \\
BMl & $\left(\mathrm{kg} / \mathrm{m}^{2}\right)$ & $21.0 \pm 2.3$ & $21.4 \pm 2.8$ & $20.5 \pm 1.4$ \\
HbA1c & $(\%)$ & $4.9 \pm 0.2$ & $4.91 \pm 0.23$ & $4.91 \pm 0.25$ \\
Hs-CRP & $(\mathrm{mg} / \mathrm{dL})$ & $0.04 \pm 0.02$ & $0.05 \pm 0.02$ & $0.03 \pm 0.02^{\S}$ \\
Adiponectin & $(\mu \mathrm{g} / \mathrm{mL})$ & $5.53 \pm 3.13$ & $4.51 \pm 2.38$ & $6.93 \pm 3.54^{\ddagger}$ \\
LPL mass & $(\mathrm{ng} / \mathrm{mL})$ & $44.1 \pm 12.7$ & $42.3 \pm 12.1$ & $48.8 \pm 15.3$ \\
\hline BMl body mass & &
\end{tabular}

BMI, body mass index; HbA1c, glycated hemoglobin A1c; Hs-CRP, high-sensitivity $\mathrm{C}$-reactive protein; $\mathrm{LPL}$, lipoprotein lipase. Values are means $\pm \mathrm{SD}$. Adiponectin values were transformed into logarithmic values. ${ }^{*} p<0.0001,{ }^{\dagger} p<0.001,{ }^{\ddagger} p<0.01$, ${ }^{\S} p<0.05$ vs. men (Welch's $t$-test). All data were derived from 45 subjects (men 26 , women 19) except LPL mass (total 21, men 15, women 6). differed between men and women, we also compared these data in Table 2B (men) and C (women).

The concentrations of all parameters were within normal limits or at low ranges during the fasting period (time 0). Fasting values of $\mathrm{TG}^{*}$, RemL-C*, RLP-C $C^{\dagger}, \mathrm{RLP}-$ $\mathrm{TG}^{\dagger}$, non-HDL-C/HDL-C*, LDL-C/HDL-C* and apoB/ apoA-I* were significantly greater in men than in women ( ${ }^{*} p<0.05 ;{ }^{\dagger} p<0.005$, Welch's $t$-test). Fasting RLP-TG concentrations in all women were undetectable $(<15 \mathrm{mg} / \mathrm{dL})$, and we set fasting RLP-TG concentration as $15 \mathrm{mg} / \mathrm{dL}$ in Table $2 \mathrm{C}$. On the other hand, HDL-C concentrations were significantly lower in men than in women ( $p<0.05$, Welch's $t$-test).

Serum concentrations of TG, RLP-C, RLP-TG, insulin and plasma glucose concentrations were significantly elevated after, compared with before loading in all participants (Table 2A). Serum RemL-C concentrations peaked at $1 \mathrm{~h}$ and were restored within $4 \mathrm{~h}$, but this elevation had no significance. On the other hand, TC, LDL-C, HDL-C, sd-LDL-C, oxidized LDL, apoA-I, apoA-II, apoB, apoC-II, apoC-III, and apoE concentrations were not elevated, which were consistent with our previous findings[11].

We also analyzed several atherogenic indicators and found that the values of non-HDL-C, non-HDL-C/ HDL-C, LDL-C/HDL-C, apoB/apoA-I, and non-HDL-C/ apoB were not significantly altered, whereas the value of TG/apoB was significantly elevated. Serum concentrations of TG, RLP-C, RLP-TG, TG/apoB and insulin separately analyzed in men and in women (Table 2B and $C$, respectively), were significantly elevated after, compared with before test meal loading.

The iAUC for TG, RemL-C, RLP-C, RLP-TG, and TG/ apoB, which were significantly increased after test meal loading, were significantly greater in men than in women $(p<0.05, p<0.005, p<0.005, p<0.01, p<0.05$, respectively, Welch's $t$-test).

Fasting serum values and the iAUC of each factor and parameter significantly and positively correlated in all participants (Fig. 1). Fasting serum values for TG, RemL-C, RLP-C, RLP-TG and TG/apoB in men $(n=26)$ also significantly and positively correlated with the iAUC $(\mathrm{r}=0.78, p<0.0001 ; \mathrm{r}=0.78, p<0.0001 ; \mathrm{r}=0.60$, $p<0.005 ; \mathrm{r}=0.54, p<0.005 ; \mathrm{r}=0.81, p<0.0001$, respectively). Fasting serum values for TG, RemL-C, and TG/apoB in women $(\mathrm{n}=19)$ significantly and positively correlated with the iAUC $(r=0.77, r=0.76$, $\mathrm{r}=0.79$, respectively; all $p<0.0001$ ), but relationships between fasting serum values of RLP-TG and the iAUC could not be estimated because fasting RLP-TG was undetectable in all of the women. Despite the small number of female participants, these results indicated that fasting values of RemL-C, RLP-C, RLP-TG, and TG/apoB in addition to TG are useful 
Table 2 Lipid and glucose parameters for before and 1 - $8 \mathrm{~h}$ after loading with test meal

\begin{tabular}{|c|c|c|c|c|c|c|c|}
\hline \multirow[t]{2}{*}{ A. All participants } & \multicolumn{7}{|c|}{ Elapsed time (h) } \\
\hline & & 0 & 1 & 2 & 4 & 6 & 8 \\
\hline $\mathrm{TC}$ & $(\mathrm{mg} / \mathrm{dL})$ & $182.4 \pm 32.8$ & $180.2 \pm 32.8$ & $178.2 \pm 31.3$ & $180.4 \pm 30.1$ & $184.0 \pm 31.6$ & $188.2 \pm 32.5$ \\
\hline LDL-C & $(\mathrm{mg} / \mathrm{dL})$ & $98.5 \pm 26.1$ & $96.3 \pm 25.5$ & $95.6 \pm 25.5$ & $96.7 \pm 24.7$ & $99.8 \pm 25.6$ & $102.1 \pm 26.2$ \\
\hline $\mathrm{HDL}-\mathrm{C}$ & $(\mathrm{mg} / \mathrm{dL})$ & $70.1 \pm 15.4$ & $68.3 \pm 15.4$ & $66.2 \pm 14.1$ & $68.3 \pm 15.0$ & $70.2 \pm 15.7$ & $72.0 \pm 16.0$ \\
\hline Sd-LDL-C & $(\mathrm{mg} / \mathrm{dL})$ & $22.4 \pm 12.1$ & $19.8 \pm 8.8$ & $18.8 \pm 8.5$ & $18.6 \pm 7.1$ & $18.6 \pm 7.1$ & $19.0 \pm 7.0$ \\
\hline OxLDL & $(\mathrm{U} / \mathrm{mL})$ & $7.38 \pm 4.96$ & $7.04 \pm 4.98$ & $7.25 \pm 5.74$ & $7.41 \pm 5.53$ & $7.71 \pm 5.41$ & $8.38 \pm 5.46$ \\
\hline TG & $(\mathrm{mg} / \mathrm{dL})$ & $69.8 \pm 31.2$ & $92.1 \pm 41.0^{*}$ & $103.9 \pm 51.4^{\dagger}$ & $72.9 \pm 40.4$ & $70.7 \pm 34.3$ & $53.9 \pm 23.8$ \\
\hline Non-HDL-C & $(\mathrm{mg} / \mathrm{dL})$ & $112.3 \pm 30.1$ & $112.0 \pm 29.9$ & $112.0 \pm 29.3$ & $112.0 \pm 28.4$ & $113.8 \pm 28.7$ & $116.3 \pm 29.4$ \\
\hline RemL-C & $(\mathrm{mg} / \mathrm{dL})$ & $3.51 \pm 1.84$ & $4.35 \pm 2.11$ & $4.29 \pm 2.46$ & $3.90 \pm 2.38$ & $3.22 \pm 1.61$ & $2.98 \pm 1.40$ \\
\hline RLP-C & $(\mathrm{mg} / \mathrm{dL})$ & $3.20 \pm 1.30$ & $4.22 \pm 1.91^{*}$ & $5.01 \pm 2.42^{\ddagger}$ & $3.88 \pm 1.94$ & $3.03 \pm 1.02$ & $2.82 \pm 0.88$ \\
\hline RLP-TG & $(\mathrm{mg} / \mathrm{dL})$ & $16.3 \pm 4.0$ & $25.5 \pm 14.4^{\dagger}$ & $32.6 \pm 21.8^{\ddagger}$ & $21.1 \pm 12.3$ & $15.8 \pm 3.4$ & $15.3 \pm 2.0$ \\
\hline ApoA-I & $(\mathrm{mg} / \mathrm{dL})$ & $161.2 \pm 26.6$ & $159.8 \pm 27.3$ & $158.7 \pm 25.2$ & $160.3 \pm 25.2$ & $163.3 \pm 26.4$ & $165.7 \pm 27.0$ \\
\hline ApoA-II & $(\mathrm{mg} / \mathrm{dL})$ & $37.6 \pm 6.0$ & $37.3 \pm 6.2$ & $36.8 \pm 6.0$ & $37.4 \pm 5.9$ & $37.7 \pm 5.9$ & $38.4 \pm 6.1$ \\
\hline ApoB & $(\mathrm{mg} / \mathrm{dL})$ & $66.6 \pm 15.3$ & $65.7 \pm 15.1$ & $65.0 \pm 14.5$ & $66.2 \pm 14.5$ & $67.9 \pm 14.8$ & $69.3 \pm 15.2$ \\
\hline ApoC-II & $(\mathrm{mg} / \mathrm{dL})$ & $3.2 \pm 1.2$ & $3.3 \pm 1.2$ & $3.3 \pm 1.2$ & $3.3 \pm 1.2$ & $3.3 \pm 1.1$ & $3.3 \pm 1.1$ \\
\hline ApoC-III & $(\mathrm{mg} / \mathrm{dL})$ & $9.5 \pm 2.4$ & $9.8 \pm 2.5$ & $9.4 \pm 2.3$ & $9.2 \pm 2.2$ & $9.0 \pm 2.1$ & $9.0 \pm 2.2$ \\
\hline ApoE & $(\mathrm{mg} / \mathrm{dL})$ & $4.3 \pm 1.1$ & $4.3 \pm 1.1$ & $4.3 \pm 1.0$ & $4.2 \pm 1.1$ & $4.1 \pm 1.0$ & $4.2 \pm 1.1$ \\
\hline Non-HDL-C/HDL-C & & $1.70 \pm 0.73$ & $1.75 \pm 0.75$ & $1.80 \pm 0.75$ & $1.75 \pm 0.76$ & $1.73 \pm 0.74$ & $1.72 \pm 0.73$ \\
\hline LDL-C/HDL-C & & $1.50 \pm 0.60$ & $1.50 \pm 0.61$ & $1.52 \pm 0.61$ & $1.50 \pm 0.61$ & $1.51 \pm 0.60$ & $1.50 \pm 0.60$ \\
\hline ApoB/apoA-I & & $0.43 \pm 0.13$ & $0.42 \pm 0.13$ & $0.42 \pm 0.13$ & $0.43 \pm 0.13$ & $0.43 \pm 0.13$ & $0.43 \pm 0.13$ \\
\hline Non-HDL-C/apo B & & $1.67 \pm 0.10$ & $1.69 \pm 0.10$ & $1.71 \pm 0.11$ & $1.68 \pm 0.10$ & $1.67 \pm 0.10$ & $1.67 \pm 0.10$ \\
\hline TG/apoB & & $1.06 \pm 0.44$ & $1.41 \pm 0.56^{\S}$ & $1.61 \pm 0.71^{\neq}$ & $1.25 \pm 0.61$ & $0.93 \pm 0.40$ & $0.79 \pm 0.32$ \\
\hline Plasma glucose & & $(\mathrm{mg} / \mathrm{dL})$ & $89.9 \pm 5.7$ & $96.9 \pm 20.0^{\S}$ & $87.5 \pm 10.8$ & $87.6 \pm 4.9$ & $88.1 \pm 5.7$ \\
\hline Insulin & & $(\mu \mathrm{U} / \mathrm{mL})$ & $5.6 \pm 2.4$ & $42.4 \pm 24.6^{\ddagger}$ & $18.5 \pm 14.1^{\ddagger}$ & $5.0 \pm 2.2$ & $4.2 \pm 2.1$ \\
\hline \multicolumn{8}{|l|}{ B. Men } \\
\hline $\mathrm{TC}$ & & $(\mathrm{mg} / \mathrm{dL})$ & $183.1 \pm 33.8$ & $181.4 \pm 34.5$ & $180.0 \pm 33.3$ & $182.4 \pm 32.5$ & $185.0 \pm 33.2$ \\
\hline LDL-C & & $(\mathrm{mg} / \mathrm{dL})$ & $101.1 \pm 28.2$ & $99.0 \pm 27.5$ & $99.3 \pm 27.7$ & $99.8 \pm 26.9$ & $102.7 \pm 27.8$ \\
\hline $\mathrm{HDL}-\mathrm{C}$ & & $(\mathrm{mg} / \mathrm{dL})$ & $66.3 \pm 16.7$ & $64.7 \pm 17.4$ & $62.5 \pm 15.5$ & $65.1 \pm 17.2$ & $66.5 \pm 17.4$ \\
\hline Sd-LDL-C & & $(\mathrm{mg} / \mathrm{dL})$ & $23.9 \pm 11.0$ & $22.2 \pm 10.2$ & $21.4 \pm 9.5$ & $20.4 \pm 9.0$ & $19.8 \pm 8.0$ \\
\hline OxLDL & & $(\mathrm{U} / \mathrm{mL})$ & $7.98 \pm 5.48$ & $7.91 \pm 5.54$ & $8.12 \pm 6.19$ & $8.32 \pm 6.11$ & $8.70 \pm 6.22$ \\
\hline TG & & $(\mathrm{mg} / \mathrm{dL})$ & $78.9 \pm 36.0$ & $106.3 \pm 45.1$ & $119.5 \pm 58.9^{\dagger}$ & $94.8 \pm 51.2$ & $68.6 \pm 33.7$ \\
\hline Non-HDL-C & & $(\mathrm{mg} / \mathrm{dL})$ & $116.8 \pm 32.5$ & $116.8 \pm 32.2$ & $117.5 \pm 31.8$ & $117.3 \pm 31.1$ & $118.5 \pm 31.2$ \\
\hline RemL-C & & $(\mathrm{mg} / \mathrm{dL})$ & $4.08 \pm 2.13$ & $4.99 \pm 2.42$ & $5.16 \pm 2.81$ & $4.69 \pm 2.80$ & $3.67 \pm 1.91$ \\
\hline RLP-C & & $(\mathrm{mg} / \mathrm{dL})$ & $3.62 \pm 1.51$ & $5.00 \pm 2.03^{*}$ & $5.82 \pm 2.76^{\ddagger}$ & $4.53 \pm 2.23$ & $3.31 \pm 1.13$ \\
\hline RLP-TG & & $(\mathrm{mg} / \mathrm{dL})$ & $17.3 \pm 5.1$ & $30.0 \pm 16.7^{\S}$ & $37.8 \pm 26.2^{\ddagger}$ & $24.7 \pm 15.2$ & $16.4 \pm 4.5$ \\
\hline ApoA-I & & $(\mathrm{mg} / \mathrm{dL})$ & $155.7 \pm 29.2$ & $154.4 \pm 29.9$ & $154.3 \pm 28.7$ & $155.2 \pm 29.0$ & $157.9 \pm 29.4$ \\
\hline ApoA-\| & & $(\mathrm{mg} / \mathrm{dL})$ & $33.8 \pm 6.7$ & $38.7 \pm 6.8$ & $38.4 \pm 6.6$ & $38.8 \pm 6.5$ & $39.1 \pm 6.7$ \\
\hline ApoB & & $(\mathrm{mg} / \mathrm{dL})$ & $68.8 \pm 16.3$ & $68.3 \pm 16.3$ & $67.5 \pm 15.6$ & $68.8 \pm 15.8$ & $70.5 \pm 16.0$ \\
\hline ApoC- II & & $(\mathrm{mg} / \mathrm{dL})$ & $3.5 \pm 1.1$ & $3.6 \pm 1.1$ & $3.6 \pm 1.2$ & $3.6 \pm 1.2$ & $3.6 \pm 1.1$ \\
\hline ApoC-III & & $(\mathrm{mg} / \mathrm{dL})$ & $9.7 \pm 2.6$ & $10.2 \pm 2.8$ & $9.8 \pm 2.7$ & $9.5 \pm 2.5$ & $9.2 \pm 2.3$ \\
\hline ApoE & & $(\mathrm{mg} / \mathrm{dL})$ & $4.1 \pm 1.1$ & $4.1 \pm 1.1$ & $4.2 \pm 1.0$ & $4.0 \pm 1.1$ & $3.9 \pm 1.0$ \\
\hline Non-HDL-C/HDL-C & & $1.90 \pm 0.85$ & $1.96 \pm 0.88$ & $2.02 \pm 0.87$ & $1.96 \pm 0.89$ & $1.94 \pm 0.86$ & $1.92 \pm 0.86$ \\
\hline LDL-C/HDL-C & & $1.64 \pm 0.71$ & $1.66 \pm 0.72$ & $1.70 \pm 0.70$ & $1.66 \pm 0.72$ & $1.67 \pm 0.71$ & $1.66 \pm 0.71$ \\
\hline ApoB/apoA-I & & $0.46 \pm 0.15$ & $0.46 \pm 0.15$ & $0.46 \pm 0.15$ & $0.46 \pm 0.16$ & $0.46 \pm 0.15$ & $0.46 \pm 0.15$ \\
\hline
\end{tabular}


Table 2 Lipid and glucose parameters for before and 1 - $8 \mathbf{~ h}$ after loading with test meal (Continued)

\begin{tabular}{|c|c|c|c|c|c|c|c|}
\hline Non-HDL-C/apo B & & $1.69 \pm 0.10$ & $1.70 \pm 0.11$ & $1.73 \pm 0.12$ & $1.70 \pm 0.11$ & $1.67 \pm 0.10$ & $1.68 \pm 0.11$ \\
\hline TG/apoB & & $1.17 \pm 0.52$ & $1.58 \pm 0.63$ & $1.79 \pm 0.84^{\dagger}$ & $1.41 \pm 0.73$ & $1.00 \pm 0.49$ & $0.86 \pm 0.39$ \\
\hline Plasma glucose & $(\mathrm{mg} / \mathrm{dL})$ & $91.1 \pm 5.9$ & $97.7 \pm 19.9$ & $85.7 \pm 9.7$ & $89.2 \pm 4.6$ & $88.7 \pm 5.9$ & $87.7 \pm 5.9$ \\
\hline Insulin & $(\mu U / m L)$ & $5.0 \pm 2.1$ & $33.6 \pm 13.7^{\ddagger}$ & $11.6 \pm 7.4^{\dagger}$ & $4.5 \pm 2.0$ & $3.8 \pm 2.3$ & $3.7 \pm 1.8$ \\
\hline \multicolumn{8}{|l|}{ C. Women } \\
\hline $\mathrm{TC}$ & $(\mathrm{mg} / \mathrm{dL})$ & $181.4 \pm 32.3$ & $178.6 \pm 31.3$ & $175.7 \pm 29.1$ & $177.6 \pm 27.1$ & $182.7 \pm 30.0$ & $186.5 \pm 29.6$ \\
\hline LDL-C & $(\mathrm{mg} / \mathrm{dL})$ & $95.1 \pm 23.3$ & $92.6 \pm 22.6$ & $90.6 \pm 21.8$ & $92.5 \pm 21.4$ & $95.7 \pm 22.2$ & $97.7 \pm 22.1$ \\
\hline $\mathrm{HDL}-\mathrm{C}$ & $(\mathrm{mg} / \mathrm{dL})$ & $75.3 \pm 12.0$ & $73.2 \pm 10.9$ & $71.3 \pm 10.3$ & $72.8 \pm 10.4$ & $75.4 \pm 11.6$ & $77.0 \pm 11.6$ \\
\hline Sd-LDL-C & $(\mathrm{mg} / \mathrm{dL})$ & $20.3 \pm 13.6$ & $16.5 \pm 4.9$ & $15.3 \pm 5.3$ & $16.1 \pm 4.6$ & $16.8 \pm 5.5$ & $16.7 \pm 5.2$ \\
\hline OxLDL & $(\mathrm{U} / \mathrm{mL})$ & $6.55 \pm 4.15$ & $5.86 \pm 3.91$ & $6.06 \pm 4.99$ & $6.17 \pm 4.48$ & $6.37 \pm 3.83$ & $6.92 \pm 4.10$ \\
\hline $\mathrm{TG}$ & $(\mathrm{mg} / \mathrm{dL})$ & $57.3 \pm 16.8$ & $72.7 \pm 24.6$ & $82.6 \pm 28.3^{\|}$ & $63.7 \pm 17.0$ & $52.8 \pm 12.4$ & $45.2 \pm 10.3$ \\
\hline Non-HDL-C & $(\mathrm{mg} / \mathrm{dL})$ & $106.1 \pm 25.9$ & $105.4 \pm 25.8$ & $104.4 \pm 24.2$ & $104.8 \pm 23.2$ & $107.3 \pm 24.3$ & $109.5 \pm 23.9$ \\
\hline RemL-C & $(\mathrm{mg} / \mathrm{dL})$ & $2.73 \pm 0.95$ & $3.47 \pm 1.16$ & $3.11 \pm 1.13$ & $2.83 \pm 0.95$ & $2.60 \pm 0.77$ & $2.45 \pm 0.69$ \\
\hline RLP-C & $(\mathrm{mg} / \mathrm{dL})$ & $2.62 \pm 0.60$ & $3.16 \pm 1.06$ & $3.90 \pm 1.22^{\ddagger}$ & $2.98 \pm 0.88$ & $2.66 \pm 0.72$ & $2.52 \pm 0.54$ \\
\hline RLP-TG & $(\mathrm{mg} / \mathrm{dL})$ & 15 & $19.4 \pm 7.0$ & $25.5 \pm 11.0^{\ddagger}$ & $16.2 \pm 2.1$ & $15.0 \pm 0.2$ & 15 \\
\hline ApoA-I & $(\mathrm{mg} / \mathrm{dL})$ & $168.7 \pm 21.0$ & $167.3 \pm 21.8$ & $164.8 \pm 18.5$ & $167.3 \pm 17.3$ & $170.7 \pm 20.1$ & $172.7 \pm 20.3$ \\
\hline ApoA-II & $(\mathrm{mg} / \mathrm{dL})$ & $36.0 \pm 4.6$ & $35.4 \pm 4.7$ & $34.6 \pm 4.4$ & $35.3 \pm 4.3$ & $35.8 \pm 4.1$ & $36.5 \pm 4.5$ \\
\hline ApoB & $(\mathrm{mg} / \mathrm{dL})$ & $63.7 \pm 13.6$ & $62.2 \pm 12.9$ & $61.5 \pm 12.5$ & $62.6 \pm 12.2$ & $64.4 \pm 12.6$ & $65.8 \pm 12.4$ \\
\hline ApoC-II & $(\mathrm{mg} / \mathrm{dL})$ & $2.8 \pm 1.1$ & $2.9 \pm 1.1$ & $2.9 \pm 1.1$ & $2.9 \pm 1.0$ & $2.9 \pm 1.0$ & $2.9 \pm 1.1$ \\
\hline ApoC-III & $(\mathrm{mg} / \mathrm{dL})$ & $9.1 \pm 2.0$ & $9.3 \pm 2.0$ & $8.9 \pm 1.7$ & $8.8 \pm 1.6$ & $8.6 \pm 1.8$ & $8.7 \pm 1.8$ \\
\hline ApoE & $(\mathrm{mg} / \mathrm{dL})$ & $4.7 \pm 1.0$ & $4.6 \pm 1.1$ & $4.5 \pm 1.1$ & $4.5 \pm 1.1$ & $4.5 \pm 1.1$ & $4.6 \pm 1.1$ \\
\hline Non-HDL-C/HDL-C & & $1.43 \pm 0.38$ & $1.46 \pm 0.40$ & $1.48 \pm 0.39$ & $1.47 \pm 0.40$ & $1.45 \pm 0.39$ & $1.45 \pm 0.37$ \\
\hline LDL-C/HDL-C & & $1.28 \pm 0.33$ & $1.28 \pm 0.33$ & $1.29 \pm 0.33$ & $1.29 \pm 0.34$ & $1.29 \pm 0.33$ & $1.29 \pm 0.32$ \\
\hline ApoB/apoA-I & & $0.38 \pm 0.08$ & $0.37 \pm 0.08$ & $0.38 \pm 0.08$ & $0.38 \pm 0.08$ & $0.38 \pm 0.08$ & $0.38 \pm 0.08$ \\
\hline Non-HDL-C/apo B & & $1.66 \pm 0.11$ & $1.68 \pm 0.09$ & $1.69 \pm 0.11$ & $1.67 \pm 0.08$ & $1.66 \pm 0.10$ & $1.66 \pm 0.10$ \\
\hline TG/apoB & & $0.92 \pm 0.26$ & $1.17 \pm 0.32^{\ddagger}$ & $1.36 \pm 0.40^{\dagger}$ & $1.04 \pm 0.29$ & $0.84 \pm 0.22$ & $0.70 \pm 0.17$ \\
\hline Plasma glucose & (mg/dL) & $88.3 \pm 5.2$ & $95.8 \pm 20.6$ & $89.9 \pm 12.1$ & $85.5 \pm 5.0$ & $87.1 \pm 5.1$ & $86.5 \pm 5.7$ \\
\hline Insulin & $(\mu \mathrm{U} / \mathrm{mL})$ & $6.5 \pm 2.6$ & $54.1 \pm 31.1^{\ddagger}$ & $27.9 \pm 15.7^{\ddagger}$ & $5.6 \pm 2.4$ & $4.8 \pm 1.8$ & $3.9 \pm 1.5$ \\
\hline
\end{tabular}

Apo, apolipoprotein; HDL-C, high-density lipoprotein cholesterol; LDL-C, low-density lipoprotein cholesterol; OxLDL, oxidized LDL; RemL-C, remnant lipoprotein cholesterol measured using "MetaboLead RemL-C"; RLP-C, remnant-like particle-cholesterol measured with "JIMRO II"; RLP-TG, remnant-like particle-triglycerides; Sd-LDL-C, small, dense-LDL cholesterol; TC, total cholesterol; TG, triglycerides. TG, RemL-C, RLP-C, RLP-TG, and TG/apoB were transformed into logarithmic values. Values are expressed as means $\pm \mathrm{SD}$. ${ }^{*} p<0.05,{ }^{\dagger} p<0.005,{ }^{\ddagger} p<0.0001,{ }^{\S} p<0.01, "$. $p<0.001$, vs. time 0 (repeated ANOVA with Dunnett's test).

markers of postprandial hyperlipidemia in men and women.

\section{Correlation between fasting adiponectin concentration and fasting lipids and their parameters}

There was no significant correlation between adiponectin and several factors (Table 3). As for LPL mass, although we had data from only 21 subjects (men 15, women 6) in the fasting period, a significant correlation with adiponectin was observed in all subjects.

We examined the correlation between fasting adiponectin concentration and fasting lipids and their parameters (Table 4). Fasting adiponectin concentration positively correlated with HDL-C and apoA-I concentrations, and negatively correlated with values of TG, RemL-C, RLP-C, RLP-TG, non-HDL-C/HDL-C, LDL-C/HDL-C, and TG/
apoB. After test meal loading, fasting adiponectin concentration positively correlated with HDL-C and apoA-I concentrations, and negatively correlated with the values of TG, RemL-C, RLP-C, RLP-TG, non-HDL-C/HDL-C, LDL-C/HDL-C, apoB/apoA-I, and TG/apoB from $1 \mathrm{~h}$ to $8 \mathrm{~h}$, fully or partially. Thus, fasting adiponectin concentration negatively correlated with postprandial elevation of lipids and their parameters.

When we examined the relationship between fasting LPL mass and lipids and their parameters before and after test meal loading, there was no significant correlation except some cases of apoC-II at $0,1,4,6$, and $8 \mathrm{~h}$ and insulin at $6 \mathrm{~h}(\mathrm{r}=-0.44, \mathrm{r}=-0.44, \mathrm{r}=-0.48, \mathrm{r}=-0.47$ $\mathrm{r}=-0.50, \mathrm{r}=-0.50$, respectively, all $\mathrm{p}<0.05)$.

We also examined the relationship between adiponectin and iAUC of lipids and their parameters. Fasting 


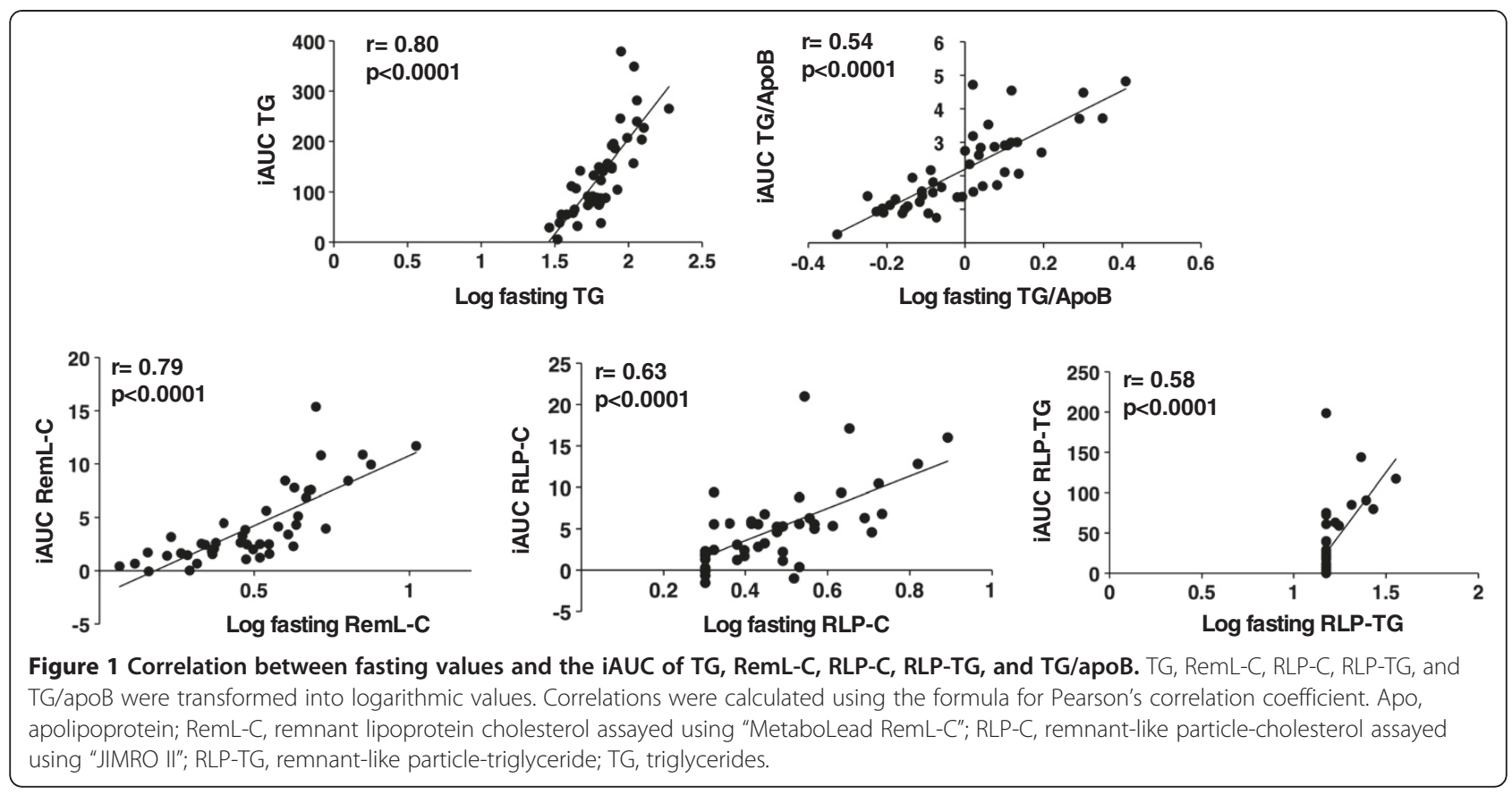

adiponectin concentration significantly and negatively correlated with the values of TG, RemL-C, RLP-C, RLPTG, and TG/apoB, but not with other factors (Fig. 2). When we examined the relationship between LPL and iAUC of them, there was no significant correlation $(\mathrm{r}=-0.02, \mathrm{p}=0.94 ; \mathrm{r}=-0.11, \mathrm{p}=0.64 ; \mathrm{r}=-0.24, \mathrm{p}=0.30$; $\mathrm{r}=-0.14, \mathrm{p}=0.56 ; \mathrm{r}=0.04, \mathrm{p}=0.87$, respectively).

\section{Multivariable linear regression analysis for postprandial TG elevation}

Because TG strongly correlated with TRL-related values such as RemL-C, RLP-C, RLP-TG, and TG/apoB $(\mathrm{r}=0.90, \mathrm{r}=0.80, \mathrm{r}=0.80, \mathrm{r}=0.83, \mathrm{p}<0.0001$, respectively), we constructed the model of multivariable linear

Table 3 Correlation between adiponectin and other characteristics

\begin{tabular}{|c|c|c|c|c|c|c|c|}
\hline & & \multicolumn{2}{|c|}{ Total } & \multicolumn{2}{|c|}{ Men } & \multicolumn{2}{|c|}{ Women } \\
\hline & & $r$ & $p$ & $r$ & $p$ & $r$ & $p$ \\
\hline Height & (m) & -0.16 & 0.31 & 0.05 & 0.83 & 0.24 & 0.33 \\
\hline Body weight & $(\mathrm{kg})$ & -0.19 & 0.20 & 0.01 & 0.96 & -0.01 & 0.97 \\
\hline Waist circumference & $(\mathrm{cm})$ & -0.26 & 0.09 & -0.08 & 0.70 & -0.21 & 0.39 \\
\hline BMI & $\left(\mathrm{kg} / \mathrm{m}^{2}\right)$ & -0.15 & 0.33 & -0.01 & 0.95 & -0.33 & 0.17 \\
\hline $\mathrm{HbA1c}$ & (\%) & -0.04 & 0.77 & 0.02 & 0.94 & -0.14 & 0.58 \\
\hline $\mathrm{Hs}-\mathrm{CRP}$ & $(\mathrm{mg} / \mathrm{dL})$ & -0.21 & 0.17 & -0.07 & 0.75 & -0.15 & 0.53 \\
\hline LPL mass & $(\mathrm{ng} / \mathrm{mL})$ & 0.54 & 0.01 & 0.34 & 0.21 & 0.81 & 0.05 \\
\hline
\end{tabular}

BMl; body mass index, HbA1c; glycated hemoglobin A1c, Hs-CRP; highsensitivity C-reactive protein, LPL; lipoprotein lipase. Adiponectin was transformed into logarithmic value. Correlation of adiponectin with other markers was analyzed by Pearson's correlation coefficient. All factors were derived from 45 subjects (men 26, women 19) except LPL mass (total 21, men 15 , women 6). regression analysis without fasting TG and adjusted by sex, BMI, age, and waist circumference as we described in Methods. RemL-C, RLP-C, RLP-TG, and TG/apoB were significant factors, but adiponectin was not (Table 5). Especially, adjusted R-squared of RemL-C and TG/apoB were 0.63 and 0.55 , respectively. Considering the correlation between fasting adiponectin and fasting lipids, we performed multivariable linear regression analysis without lipids and their parameters. However, we could not uncover a significant relationship between fasting adiponectin concentrations and postprandial TG elevation $(p=0.09)$.

\section{Discussion}

The present study demonstrated that fasting TRLrelated values of TG, RemL-C, RLP-C, RLP-TG, and TG/apoB were useful tools for predicting postprandial hyperlipidemia and that fasting adiponectin concentrations correlated with the fasting values of these lipids and parameters in young healthy individuals, although adiponectin was not a significant predictor in multivariable linear regression analysis.

Many studies have examined the relationship between adiponectin and dyslipidemia. Baratta et al. reported that the relationship between adiponectin and fasting lipid values is independent of body fat mass [21]. Kazumi et al. and Heliövaara et al. demonstrated that hypoadiponectinemia is more closely related to adiposity and fasting dyslipidemia than insulin resistance in young healthy men [22,23]. Interestingly, Yoshida et al. demonstrated the lower adiponectin concentrations in type IIb hyperlipidemia compared to 
Table 4 Correlation between adiponectin and parameters concerning lipid and glucose metabolism before and after loading the test meal

\begin{tabular}{|c|c|c|c|c|c|c|c|}
\hline & & 0 & $1 \mathrm{~h}$ & $2 \mathrm{~h}$ & $4 \mathrm{~h}$ & $6 \mathrm{~h}$ & $8 \mathrm{~h}$ \\
\hline & & $r$ & $r$ & $r$ & $r$ & $r$ & $r$ \\
\hline $\mathrm{TC}$ & $(\mathrm{mg} / \mathrm{dL})$ & 0.09 & 0.08 & 0.06 & 0.05 & 0.08 & 0.06 \\
\hline LDL-C & $(\mathrm{mg} / \mathrm{dL})$ & -0.06 & -0.06 & -0.07 & -0.09 & -0.08 & -0.09 \\
\hline $\mathrm{HDL}-\mathrm{C}$ & (mg/dL) & $0.43^{*}$ & $0.43^{*}$ & $0.45^{*}$ & $0.42^{*}$ & $0.41^{*}$ & $0.41^{* * *}$ \\
\hline Sd-LDL-C & (mg/dL) & -0.11 & -0.24 & -0.26 & -0.26 & -0.18 & -0.16 \\
\hline OxLDL & $(\mathrm{U} / \mathrm{mL})$ & 0.03 & 0.06 & 0.05 & 0.08 & 0.02 & 0.03 \\
\hline TG & (mg/dL) & $-0.42^{* * *}$ & $-0.39 * * *$ & $-0.40^{* * *}$ & $-0.48^{*}$ & $-0.40^{* * *}$ & $-0.42^{*}$ \\
\hline Non-HDL-C & (mg/dL) & -0.12 & -0.13 & -0.15 & -0.17 & -0.13 & -0.15 \\
\hline RemL-C & (mg/dL) & $-0.35^{* * * *}$ & $-0.30^{* * * *}$ & $-0.37^{* * * *}$ & $-0.44^{* * *}$ & $-0.33^{* * * *}$ & $-0.34^{* * * *}$ \\
\hline RLP-C & (mg/dL) & $-0.38^{* * * *}$ & $-0.36^{* * * *}$ & $-0.39^{* * *}$ & $-0.45^{* * *}$ & -0.28 & $-0.35^{* * * *}$ \\
\hline RLP-TG & $(\mathrm{mg} / \mathrm{dL})$ & $-0.35^{* *}$ & $-0.30^{* * * *}$ & $-0.36^{* * * *}$ & $-0.42^{*}$ & $-0.38^{* * * *}$ & $-0.34^{* * * *}$ \\
\hline ApoA-I & (mg/dL) & $0.34^{* * * *}$ & $0.37^{* * * *}$ & $0.35^{* * * *}$ & $0.33^{* * * *}$ & $0.34^{* * * *}$ & $0.35^{* * * *}$ \\
\hline ApoA-II & (mg/dL) & -0.06 & -0.07 & -0.11 & -0.11 & -0.07 & -0.07 \\
\hline ApoB & (mg/dL) & -0.09 & -0.10 & -0.13 & -0.13 & -0.12 & -0.13 \\
\hline ApoC-II & $(\mathrm{mg} / \mathrm{dL})$ & -0.29 & -0.27 & -0.27 & $-0.32^{* * * *}$ & -0.27 & -0.24 \\
\hline ApoC-III & (mg/dL) & -0.21 & -0.18 & -0.21 & -0.27 & -0.20 & -0.13 \\
\hline ApoE & (mg/dL) & 0.06 & 0.06 & 0.03 & 0.02 & 0.08 & 0.10 \\
\hline Non-HDL-C/HDL-C & & $-0.37^{* * * *}$ & $-0.38^{* * *}$ & $-0.38^{* * *}$ & $-0.38^{* * *}$ & $-0.37^{* * * *}$ & $-0.38^{* * *}$ \\
\hline LDL-C/HDL-C & & $-0.34^{* * * *}$ & $-0.35^{* * * *}$ & $-0.35^{* * * *}$ & $-0.35^{* * * *}$ & $-0.35^{* * * *}$ & $-0.36^{* * * *}$ \\
\hline ApoB/ApoA-I & & -0.29 & $-0.31^{* * * *}$ & $-0.31^{* * * *}$ & -0.30 & $-0.31^{* * * *}$ & $-0.32^{* * * *}$ \\
\hline Non-HDL-C/ApoB & & -0.21 & -0.21 & -0.14 & -0.26 & -0.11 & -0.14 \\
\hline TG/apoB & & $-0.40^{* * *}$ & $-0.39 * * *$ & $-0.37^{* * * *}$ & $-0.43^{* * *}$ & $-0.33^{* * * *}$ & $-0.35^{* * * *}$ \\
\hline Plasma glucose & $x(m g / d L)$ & -0.12 & -0.06 & -0.04 & -0.13 & -0.12 & -0.11 \\
\hline Insulin & $(\mu \mathrm{U} / \mathrm{mL})$ & -0.17 & -0.02 & -0.07 & -0.22 & $-0.33^{* * * *}$ & -0.22 \\
\hline
\end{tabular}

TC; total cholesterol, LDL-C; low-density lipoprotein-cholestrol, HDL-C; high-density lipoprotein-cholesterol, Sd-LDL; small, dense-LDL, OxLDL; oxidized LDL, TG; triglycerides, RemL-C; remnant lipoprotein cholesterol measured with "MetaboLead RemL-C", RLP-C; remnant-like particle-cholesterol measured with "JIMRO II", RLP-TG; remnant-like particle-triglycerides, Apo; apolipoprotein. TG, RemL-C, RLP-C, RLP-TG, TG/apoB and adiponectin were transformed into logarithmic values. Correlation of adiponectin with other markers was analyzed by Pearson's correlation coefficient. ${ }^{*} p<0.005,{ }^{* *} p<0.001,{ }^{* * *} p<0.01,{ }^{* * * *} p<0.05$.

normolipidemia, type IV, and type IIa hyperlipidemia in type II diabetes patients [24]. On the other hand, the relationship between adiponectin and postprandial hyperlipidemia has not been investigated in detail. Rubin et al. demonstrated that postprandial plasma adiponectin values decreased after oral loading with $75 \mathrm{~g}$ of glucose or with a high-fat meal (58 $\mathrm{g}$ of fat) and that postprandial TG concentrations correlated with fasting adiponectin concentration in a 45- to 65year-old population including those with metabolic syndrome [16]. Moreover, Maruyama et al. found lower TG and RLP-TG concentrations among individuals with type 1 diabetes and a high, rather than a low baseline HMW adiponectin concentration at baseline and at $3 \mathrm{~h}$ after test meal loading [17]. The present study demonstrated that fasting adiponectin concentration positively correlated with fasting $\mathrm{HDL}-\mathrm{C}$ and apoA-I concentrations, and negatively correlated with fasting and postprandial values of TG, RemL-C, RLPC, RLP-TG, non-HDL-C/HDL-C, LDL-C/HDL-C and TG/apoB. These are new findings compared with our previous study [11]. However, multivariable linear regression analysis showed that adiponectin was not significant for predicting postprandial hyperlipidemia, which is difficult to explain. Adiponectin participates in the metabolism of the visceral fat mass, glucose and lipids, but it might not reflect dynamic changes such as lipid concentrations that elevate after test meal loading. In addition, the small number of samples derived from only healthy subjects might have affected this finding. Patients with hyperlipidemia, diabetes or metabolic syndrome often have delayed TRL clearance [10]. Although we may find other results if we include older subjects or these patients, it is also necessary to elucidate the significant predictive factors in young healthy subjects, because our purpose of the present 


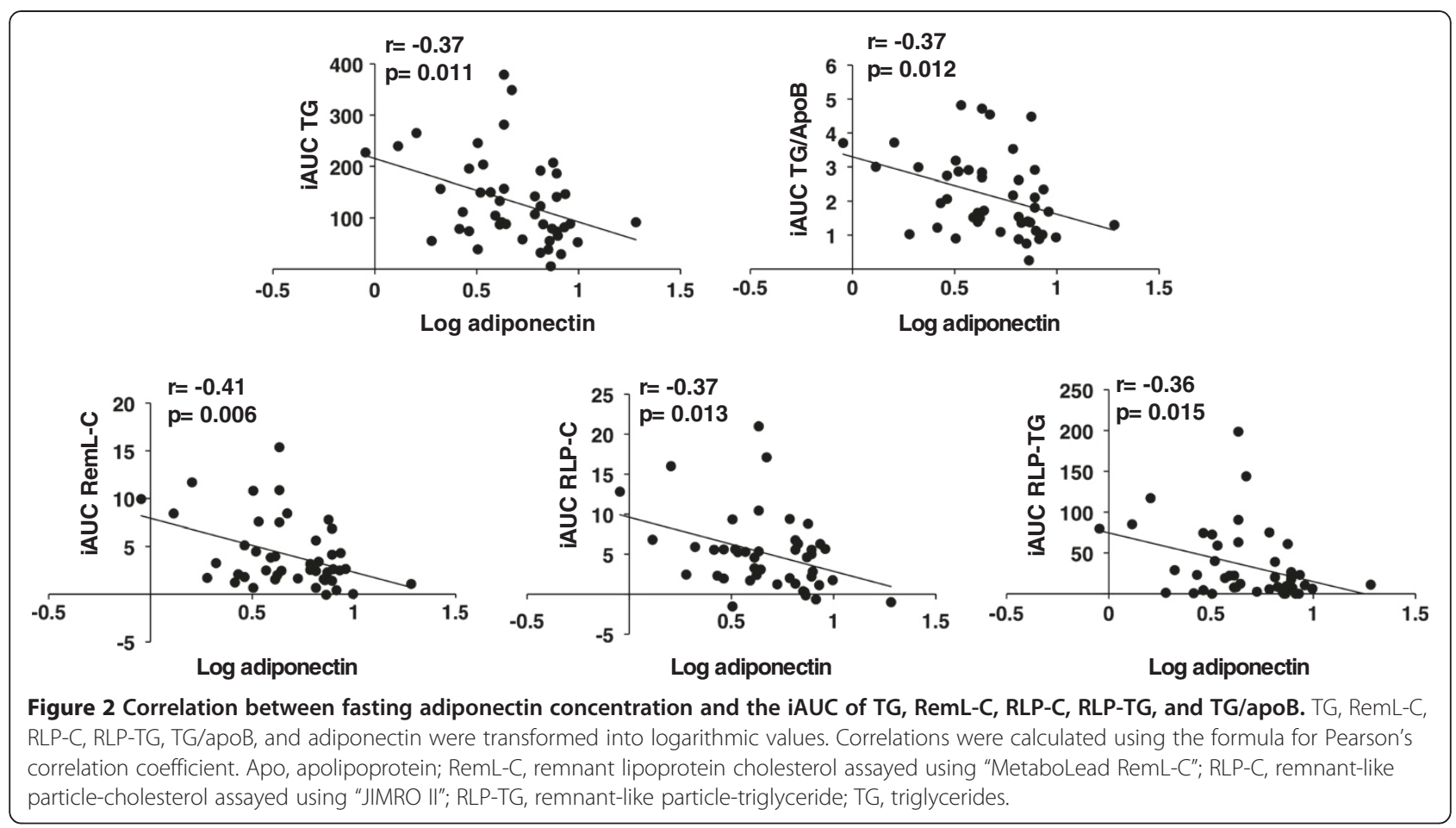

study is to elucidate the significant predictors of postprandial TG elevation. On the other hand, since adiponectin concentrations are considerably higher in women than in men, the significance of adiponectin needs to be separately analyzed in men and women.

A definition or standard method other than TG elevation has not been established for predicting postprandial hyperlipidemia. This complicates resolving which factor among those associated with postprandial hyperlipidemia is the most useful for predicting CVD. Some studies have demonstrated the superiority of non-fasting, over fasting TG concentrations for predicting CVD [6,7]. Oka et al. notably demonstrated that waist circumference is more closely related to postprandial, than to fasting TG [25]. Moreover, the lipid profile in metabolic syndrome includes elevated TG and remnant lipoproteins, de creased LDL particle size and low HDL-C concentrations [10]. Thus, not only fasting TG values, but also other parameters might be required to assess CVD risk. Remnant lipoproteins play an important role in atherogenesis and their concentrations are useful to understand metabolic disorders and to predict CVD [10,26,27]. Ai et al. reported that RLP-C and RLP-TG, but not the TG response to an oral fat load are significantly increased in hyperinsulinemic patients with type 2 diabetes [28]. We also found that fasting serum concentrations of remnant lipoproteins might be useful to detect postprandial hyperlipidemia even in normolipidemic individuals [11]. Here, we confirmed that remnant lipoproteins during the fasting period can predict postprandial hyperlipidemia. The purpose of our study was to elucidate the factors that predict postprandial hyperlipidemia besides TG, because

Table 5 Multivariable linear regression analysis for postprandial TG elevation (adjusted by sex, BMI, age, and waist circumference)

\begin{tabular}{|c|c|c|c|c|c|c|}
\hline & $\beta$ Coefficient & SE & $95 \% \mathrm{Cl}$ & $p$ & $\begin{array}{l}\text { Standardized partial } \\
\text { regression coefficient }\end{array}$ & $\begin{array}{l}\text { Adjusted } \\
\text { R-squared }\end{array}$ \\
\hline RemL-C & 0.67 & 0.09 & 0.48 to 0.86 & $<0.01$ & 0.72 & 0.63 \\
\hline RLP-C & 0.71 & 0.17 & 0.37 to 1.06 & $<0.01$ & 0.55 & 0.41 \\
\hline RLP-TG & 1.21 & 0.36 & 0.48 to 1.93 & $<0.01$ & 0.48 & 0.33 \\
\hline TG/apoB & 0.81 & 0.13 & 0.54 to 1.08 & $<0.01$ & 0.66 & 0.55 \\
\hline Adiponectin & -0.21 & 0.12 & -0.45 to 0.03 & 0.09 & -0.27 & 0.20 \\
\hline
\end{tabular}

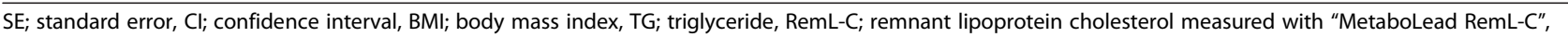
RLP-C; remnant-like particle-cholesterol measured with "JIMRO II", RLP-TG; remnant-like particle-triglycerides, Apo; apolipoprotein. RemL-C, RLP-C, RLP-TG, TG/apoB and adiponectin were transformed into logarithmic values. 
it is difficult to select high-risk patients with TG alone. However, TG strongly correlated with TRL-related values including RemL-C, RLP-C, RLP-TG, and TG/apoB, so we constructed the regression model without fasting $\mathrm{TG}$. Multivariable linear regression analysis identified RemLC, RLP-C, RLP-TG, and TG/apoB as significant predictors of postprandial TG elevation. Taking these findings together, these TRL-related values might be clinically useful for predicting postprandial hyperlipidemia.

Another novel outcome of the present study is that the amount of postprandial lipid elevation differs between men and women. Fasting values of TG, RemL-C, RLP-C, RLP-TG, non-HDL-C/HDL-C, LDL-C/HDL-C, and apoB/apoA-I were significantly greater in men than in women (Table $2 \mathrm{~B}$ and $\mathrm{C}$ ). Furthermore, the iAUCs of TG, RemL-C, RLP-C, RLP-TG, and TG/apoB were also significantly greater in men than in women. These results suggest that men are more susceptible to postprandial hyperlipidemia. The difference in lipoprotein metabolism between men and women may be caused by several mechanisms including lipoprotein lipase and lecithincholesterol acyltransferase activities (LCAT) activities, and gender specific hormonal effects [8-10,29]. Higher adiponectin concentrations in women may also influence TRL metabolism (Table 1) [14,15,21-24]. Although we did not check the activities of enzymes and menstrual cycle of each woman in the present study, these differences in fasting and postprandial TRL-related lipid concentrations between men and women should be considered when distinguishing and predicting individuals at high-risk for postprandial hyperlipidemia.

We measured the serum concentrations of several lipid markers. Postprandial accumulation of TRL was strongly associated with the increased prevalence of sd-LDL in patients with myocardial infarction [30]. However, sd-LDL concentrations were decreased in the present study of young healthy individuals. Ogita et al. demonstrated that serum sd-LDL concentrations decrease after meals, increase during the night and peak just before breakfast [31]. Hirayama et al. also demonstrated a similar decrease in the sd-LDL concentrations after breakfast; they speculated that sd-LDL permeates the vascular walls more easily, and might be more susceptible than buoyant LDL to entrapment in vascular subendothelial spaces [32]. The precise mechanism should be addressed in future studies. Regardless, postprandial changes in sd-LDL might differ between healthy individuals and patients with CVD.

We also found that the oxidized LDL concentration did not significantly change. Although oxidized LDL in patients with CAD is postprandially elevated [33], concentrations in healthy individuals have not been investigated in detail. One study has found that oxidized LDL concentrations are not elevated in individuals with normal glucose tolerance during oral glucose tolerance test [34].
Postprandial changes in oxidized LDL concentrations should be examined and compared with those of patients with CAD.

Non HDL-C is an excellent predictor of atherosclerotic risk [35,36] and it is free of dietary variations $[36,37]$. Ogita et al. also demonstrated that serum concentrations of TC, HDL-C, and LDL-C do not change remarkably in healthy individuals [31]. The present study also did not identify significant changes in TC, HDL-C, LDL-C and non HDL-C concentrations among young healthy individuals. Some studies have demonstrated that LDL-C and HDL-C concentrations decrease during the day in a rhythmic circadian manner [38,39]. We also found decreased LDL-C and HDL-C concentrations, but the changes were not statistically significant. Thus, TC and non HDL-C concentrations were not significantly altered, although the postprandial value of remnant cholesterol was increased.

This study has some limitations. Although others have associated adiponectin concentrations with body weight, waist circumference, BMI, HbA1c and hs-CRP [40,41], we found no significant correlations because we measured these values in young individuals without metabolic syndrome or diabetes.

The RemL-C concentrations increased after test meal loading, albeit without significance, which is consistent with previous findings [11]. Lipoproteins targeted by both RLP-C and RemL-C include remnants of both chylomicrons and VLDL [10]. Concentrations of RemL-C and RLP-C closely correlate in patients with coronary artery disease, but the sensitivity of detecting chylomicron remnant (exogenous) and VLDL remnant (endogenous) lipoproteins might differ between analytical methods [10]. By detailed analysis with the high performance liquid chromatography method, Yoshida et al. demonstrated that they found higher concentrations of chylomicron cholesterol in serum samples with RemL-C $<$ RLP-C, but high concentrations of intermediate-density lipoprotein (IDL)cholesterol (VLDL remnant cholesterol) in samples with RemL-C > RLP-C [42]. Similarly, we and others also reported that methods for measuring RLP-C and RLP-TG might be more sensitive to chylomicron remnantcholesterol and -triglycerides, whereas those for RemL-C might be more suitable for IDL-cholesterol [11,20,43]. This may cause a difference in elevation after test meal loading and in multivariable linear regression analysis between RemL-C and RLP-C in the present study.

We did not evaluate the effect of activities such as aerobic exercise that can decrease TG and remnant concentrations and increase adiponectin concentrations [44]. Factors that can predict postprandial hyperlipidemia should be investigated in larger populations including individuals with metabolic syndrome, diabetes and CVD. 


\section{Conclusion}

Fasting TRL-related values, especially RemL-C, RLP-C, RLP-TG, and TG/apoB are useful predictors of post prandial hyperlipidemia in young healthy individuals. Although the fasting adiponectin concentration correlates with the fasting values of TG, RemL-C, RLP-C, RLP-TG and TG/apoB, it is not a significant predictor of postprandial hyperlipidemia in multivariable linear regression analysis.

\begin{abstract}
Abbreviations
TG: Triglycerides; CVD: Cardiovascular diseases; TRL: Triglyceride-rich lipoproteins; VLDL: Very-low-density lipoprotein; apo: Apolipoprotein; HMW: High molecular weight; RLP-C: Remnant-like particle-cholesterol; RLP-TG: Remnant-like particle-triglycerides; RemL-C: Remnant lipoprotein cholesterol; BMI: Body mass index; LDL-C: LOW-density lipoproteincholesterol; HDL-C: High-density lipoprotein-cholesterol; sd-LDL-C: Small dense LDL-cholesterol; hsCRP: High-sensitivity C-reactive protein; HbA1c: Glycosylated hemoglobin A1c; iAUC: Incremental area under the curve; OxLDL: Oxidized LDL; SE: Standard error; Cl: Confidence interval.
\end{abstract}

\section{Competing interests}

There is no conflict of interest for this paper.

\section{Authors' contributions}

All authors should have made substantial contributions to all of the following: (1) the conception and design of the study, or acquisition of data, or analysis and interpretation of data, (2) drafting the article or revising it critically for important intellectual content, (3) final approval of the version to be submitted. Especially, TN, ST, HO, IS, MY, HK, SK, and YF actually performed the examination according to the protocol. TN, SY, YI and YF were involved in protocol development, gaining ethical approval, and patient recruitment. TN, DS, TK and YF were involved in data analysis.

\section{Declarations}

Submission of our article implies that the work described has not been published previously that it is not under consideration for publication elsewhere, that its publication is approved by all authors and tacitly or explicitly by the responsible authorities where the work was carried out, and that, if accepted, it will not be published elsewhere in the same form, in English or in any other language, including electronically without the written consent of the copyright-holder.

\section{Acknowledgement}

This study was supported by Grant-in-Aid for Health-Science Research of Kobe Gakuin University. The study protocol was approved by the institutional ethics committee of Kobe Gakuin University (HEB080701-1) and carried out according to the Declaration of Helsinki.

\section{Author details \\ ${ }^{1}$ Division of Clinical Nutrition, Faculty of Nutrition, Kobe Gakuin University, 518 Arise, Ikawadani-cho, Nishi-ku, Kobe 651-2180, Japan. ${ }^{2}$ Section of Evidence-based Laboratory Medicine, Division of Clinical Pathology and Immunology, Department of Internal Medicine Related, Kobe University Graduate School of Medicine, Kobe, Japan. ${ }^{3}$ Department of Clinical Laboratory, Kobe University Hospital, Kobe, Japan. ${ }^{4}$ Department of Cardiovascular Medicine, Osaka University Graduate School of Medicine, Osaka, Japan. ${ }^{5}$ Department of Internal Medicine, Kakogawa West City Hospital, Kakogawa, Japan. ${ }^{6}$ Division of Clinical Laboratory, Shimane Prefectural Central Hospital, Shimane, Japan. ${ }^{7}$ Department of Preventive Medicine and Public Health, School of Medicine, Keio University, Tokyo, Japan. ${ }^{8}$ Division of Nutrition Guidance, Kakogawa East City Hospital, Kakogawa, Japan.}

Received: 6 September 2012 Accepted: 26 October 2012 Published: 31 October 2012

\section{References}

1. Miller M, Stone NJ, Ballantyne C, Bittner V, Criqui MH, Ginsberg HN, Goldberg AC, Howard WJ, Jacobson MS, Kris-Etherton PM, Lennie TA, Levi M, Mazzone T, Pennathur S: on behalf of the American Heart Association Clinical Lipidology, Thrombosis, and Prevention Committee of the Council on Nutrition, Physical Activity, and Metabolism, Council on Arteriosclerosis, Thrombosis and Vascular Biology, Council on Cardiovascular N: Triglycerides and cardiovascular disease. A Scientific Statement From the American Heart Association. Circulation 2011, 123:2292-2333.

2. Iso H, Naito $Y$, Sato $S$, Kitamura A, Okamura T, Sankai T, Shimamoto T, lida $M$, Komachi $Y$ : Serum triglycerides and risk of coronary heart disease among Japanese men and women. Am J Epidemiol 2001, 153:490-499.

3. Eberly LE, Stamler J, Neaton JD, For the Multiple Risk Factor Intervention Trial Research Group: Relation of triglyceride levels, fasting and nonfasting, to fatal and nonfatal coronary heart disease. Arch Intern Med 2003, 163:1077-1083.

4. Zilversmit DB: Atherogenesis: a postprandial phenomenon. Circulation 1979, 60:473-485.

5. Patsch JR, Miesenböck G, Hopferwieser T, Mühlberger V, Knapp E, Dunn JK, Gotto AM Jr, Patsch W: Relation of triglyceride metabolism and coronary disease. Studies in the postprandial state. Arterioscler Thromb 1992, 12:1336-1345.

6. Bansal S, Buring JE, Rifai N, Mora S, Sacks FM, Ridker PM: Fasting compared with nonfasting triglycerides and risk of cardiovascular events in women. JAMA 2007, 298:309-316.

7. Nordestgaard BG, Benn M, Schnohr P, Tybjaerg-Hansen A: Nonfasting triglycerides and risk of myocardial infarction, ischemic heart disease, and death in men and women. JAMA 2007, 298:299-308.

8. Karpe F, Hamsten A: Postprandial lipoprotein metabolism and atherosclerosis. Curr Opin Lipidol 1995, 6:123-129.

9. Davignon J, Cohn JS: Triglycerides: a risk factor for coronary heart disease. Atherosclerosis 1996, 124(Suppl):S57-S64.

10. Fujioka $Y$, Ishikawa $Y$ : Remnant lipoproteins as strong key particles to atherosclerosis. J Atheroscler Thromb 2009, 16:145-154.

11. Sato I, Ishikawa Y, Ishimoto I, Katsuta S, Toyokawa A, Hayashi F, Kawano S, Fujioka Y, Yamashita S, Kumagai S: Significance of measuring serum concentrations of remnant lipoproteins and apolipoprotein B-48 in fasting period. J Atheroscler Thromb 2009, 16:12-20.

12. Maeda K, Okubo K, Shimomura I, Funahashi T, Matsuzawa Y, Matsubara K: cDNA cloning and expression of a novel adipose-specific collagen-like factor, apM1 (adipose most abundant gene transcript 1). Biochem Biophys Res Commun 1996, 221:286-289.

13. Funahashi T, Matsuzawa Y: Metabolic syndrome: clinical concept and molecular basis. Ann Med 2007, 39:482-494.

14. Chan DC, Watts GF, Ng TWK, Uchida Y, Sakai N, Yamashita S, Barrett PHR: Adiponectin and other adipocytokines as predictors of markers of triglyceride-rich lipoprotein metabolism. Clin Chem 2005, 51:578-585.

15. Cnop M, Havel PJ, Utzschneider KM, Carr DB, Sinha MK, Boyko EJ, Retzlaff BM, Knopp RH, Brunzell JD, Kahn SE: Relationship of adiponectin to body fat distribution, insulin sensitivity and plasma lipoproteins: evidence for independent roles of age and sex. Diabetologia 2003, 46:459-469.

16. Rubin D, Helwig U, Northnagel M, Lemke N, Schreiber S, Fölsch UR, Döring F, Schrezenmeir J: Postprandial plasma adiponectin decreases after glucose and high fat meal and is independently associated with postprandial triacylglycerols but not with -11388 promotor polymorphism. Br J Nutr 2008, 99:76-82.

17. Maruyama C, Ishibashi R, Araki R, Koike S, Hirose H, Maruyama T: HMWAdiponectin associates with triglyceride concentrations in type 1 diabetic patients. J Atheroscler Thromb 2009, 16:207-216.

18. Hirano $T$, Ito $Y$, Yoshino $G$ : Measurement of small dense low-density lipoprotein particles. J Atheroscler Thromb 2005, 12:67-72.

19. Nakajima K, Saito T, Tamura A, Suzuki M, Nakano T, Adachi M, Tanaka A, Tada N, Nakamura H, Campos E, Havel RJ: Cholesterol in remnant-like lipoproteins in human serum using monoclonal anti apo B-100 and anti A-I immunoaffinity mixed gel. Clin Chim Acta 1993, 223:53-71.

20. Miyauchi K, Kayahara N, Ishigami M, Kuwata H, Mori H, Sugiuchi H, Irie T, Tanaka A, Yamashita S, Yamamura T: Development of a homogeneous assay to measure remnant lipoprotein cholesterol. Clin Chem 2007, 53:2128-2135. 
21. Baratta $R$, Amato $S$, Degano $C$, Farina $M G$, Patanè $G$, Vigneri $R$, Frittitta L: Adiponectin relationship with lipid metabolism is independent of body fat mass: evidence from both cross-sectional and intervention studies. J Clin Endocrinol Metab 2004, 89:2665-2671.

22. Kazumi T, Kawaguchi A, Hirano T, Yoshino G: Serum adiponectin is associated with high-density lipoprotein cholesterol, triglycerides, and low-density lipoprotein size in young healthy men. Metabolism 2004, 53:589-593.

23. Heliövaara MK, Strandberg TE, Karonen SL, Ebeling P: Association of serum adiponectin concentration to lipid and glucose metabolism in healthy humans. Horm Metab Res 2006, 38:336-340.

24. Yoshida H, Hirowatari Y, Kurosawa H, Tada N: Implications of decreased serum adiponectin for type Ilb hyperlipidaemia and increased cholesterol levels of very-low-density lipoprotein in type II diabetic patients. Clin Sci 2005, 109:297-302.

25. Oka R, Kobayashi J, Miura K, Nagasawa S, Moriuchi T, Hifumi S, Miyamoto S, Kawaashiri MA, Nohara A, Inazu A, Takeda Y, Mabuchi H, Yagi K, Yamagishi $M$ : Difference between fasting and nonfasting triglyceridemia; the influence of waist circumference. J Atheroscler Thromb 2009, 16:633-640.

26. Kugiyama K, Doi H, Takazoe K, Kawano H, Soejima H, Mizuno Y, Tsunoda R, Sakamoto T, Nakano T, Nakajima K, Ogawa H, Sugiyama S, Yoshimura M, Yasue $\mathrm{H}$ : Remnant lipoprotein levels in fasting serum predict coronary events in patients with coronary artery disease. Circulation 1999, 99:2858-2860.

27. Nakamura T, Takano H, Umetani K, Kawabata K, Obata JE, Kitta Y, Kodama Y, Mende A, Ichigi Y, Fujioka D, Saito Y, Kugiyama K: Remnant lipoproteinemia is a risk factor for endothelial vasomotor dysfunction and coronary artery disease in metabolic syndrome. Atherosclerosis 2005, 181:321-327.

28. Ai M, Tanaka A, Ogita K, Sekine M, NumanoF NF, Reaven GM: Relation between plasma insulin concentration and plasma remnant lipoprotein response to an oral fat load in patients with type 2 diabetes. J Am Coll Cardiol 2001, 38:1628-1632.

29. Rousset X, Vaisman B, Amar M, Sethi AA, Remaley AT: Lecithin: Cholesterol acyltransferase-from biochemistry to role in cardiovascular disease. Curr Opin Endocrinol Diabetes Obes 2009, 16:163-171.

30. Tsunoda F, Koba S, Hirano T, Ban Y, Iso Y, Suzuki H, Geshi E, Katagiri T: Association between small dense low-density lipoprotein and postprandial accumulation of triglyceride-rich remnant-like particles in normotriglyceridemic patients with myocardial infarction. Circ J 2004, 68:1165-1172

31. Ogita K, Ai M, Tanaka A, Ito Y, Hirano T, Yoshino G, Shimokado K: Circadian rhythm of serum concentration of small sense low-density lipoprotein cholesterol. Clin Chim Acta 2007, 376:96-100.

32. Hirayama S, Soda S, Ito Y, Matsui H, Ueno T, Fukushima Y, Ohmura H, Hanyu $\mathrm{O}$, Aizawa $Y$, Miida T: Circadian change of serum concentration of small sense LDL-cholesterol in type 2 diabetic patients. Clin Chim Acta 2010, 411:253-257.

33. Granér M, Kahri J, Nakano T, Sarna SJ, Niemien MS, Syvänne M, Taskinen MR: Impact of postprandial lipaemia on low-density lipoprotein (LDL) size and oxidized LDL in patients with coronary artery disease. Eur J Clin Invest 2006, 36:764-770.

34. Serin O, Konukoglu D, Firtina S, Mavis O: Serum oxidized low density lipoprotein, paraoxonase 1 and lipid peroxidation levels during oral glucose tolerance test. Horm Metab Res 2007, 39:207-211.

35. Arsenault BJ, Rana JS, Stroes ES, Després JP, Shah PK, Kastelein JJ, Wareham NJ, Boekholdt SM, Khaw KT: Beyond low-density lipoprotein cholesterol: respective contributions of non-high-density lipoprotein cholesterol levels, triglycerides, and the total cholesterol/high-density lipoprotein cholesterol ratio to coronary heart disease risk in apparently healthy men and women. J Am Coll Cardiol 2009, 55:35-41.

36. Shimano H, Arai H, Harada-Shiba M, Ueshima H, Ohta T, Yamashita S, Gotoda T, Kiyohara Y, Hayashi T, Kobayashi J, Shimamoto K, Bujo H, Ishibashi S, Shirai K, Oikawa S, Saito Y, Yamada N: Proposed guidelines for hypertriglyceridemia in Japan with non-HDL cholesterol as the second target. J Atheroscler Thromb 2008, 15:116-121.

37. Lichtenstein AH, Ausman LM, Carrasco W, Jenner JL, Gualtieri LJ, Goldin BR Ordovas JM, Schaefer EJ: Effects of canola, corn, and olive oils on fasting and postprandial plasma lipoproteins in humans as part of a national cholesterol education program step 2 diet. Arterioscler Thromb 1993 13:1533-1542
38. Romon M, Le Fur C, Lebel P, Edmé JL, Fruchart JC, Dallongeville J: Circadian variation of postprandial lipemia. Am J Clin Nutr 1997, 65:934-940.

39. Miida T, Nakamura Y, Mezuki T, Hanyu O, Maruyama S, Horikawa Y, Izawa S, Yamada Y, Matsui H, Okada M: LDL-cholesterol and HDL-cholesterol concentrations decrease during the day. Ann Clin Biochem 2002, 39:241-249.

40. Mantzoros CS, Li T, Manson JE, Meigs JB, Hu FB: Circulating adiponectin levels are associated with better glycemic control, more favorable lipid profile, and reduced inflammation in women with type 2 diabetes. $J$ Clin Endocrinol Metab 2005, 90:4542-4548.

41. Fujimatsu D, Kotooka N, Inoue T, Nishiyama M, Node K: Association between high molecular weight adiponectin levels and metabolic parameters. J Atheroscler Thromb 2009, 16:553-559.

42. Yoshida H, Kurosawa H, Hirowatari Y, Ogura Y, Ikewaki K, Abe I, Saikawa S, Domitsu K, Ito K, Yanai H, Tada N: Characteristic comparison of triglyceride-rich remnant lipoprotein measurement between a new homogenous assay (RemL-C) and a conventional immunoseparation method (RLP-C). Lipids Health Dis 2008, 7:18.

43. Nakada Y, Kurosawa H, Tohyama J, Inoue Y, Ikewaki K: Increased remnant lipoprotein in patients with coronary artery disease-evaluation utilizing a newly developed remnant assay, remnant lipoproteins cholesterol homogenous assay (RemL-C). J Atheroscler Thromb 2007, 14:56-64.

44. Yoshida $H$, Ishikawa $T$, Suto M, Kurosawa $H$, Hirowatari $Y$, Ito K, Yanai H, Tada N, Suzuki M: Effects of supervised aerobic exercise training on serum adiponectin and parameters of lipid and glucose metabolism in subjects with moderate dyslipidemia. J Atheroscler Thromb 2010, 17:1160-1166.

doi:10.1186/1476-511X-11-146

Cite this article as: Nagata et al:: Fasting remnant lipoproteins can predict postprandial hyperlipidemia. Lipids in Health and Disease 2012 11:146.

\section{Submit your next manuscript to BioMed Central and take full advantage of:}

- Convenient online submission

- Thorough peer review

- No space constraints or color figure charges

- Immediate publication on acceptance

- Inclusion in PubMed, CAS, Scopus and Google Scholar

- Research which is freely available for redistribution 